\title{
Refractory Cryptococcus neoformans Meningoencephalitis in an Immunocompetent Patient: Paradoxical Antifungal Therapy-Induced Clinical Deterioration Related to an Immune Response to Cryptococcal Organisms
}

\author{
Hideto Nakajima ${ }^{a, b} \quad$ Ayami Takayama $^{a, b} \quad$ Yohei Fujiki $^{a, b} \quad$ Takumi Ito $^{a}$ \\ Haruko Kitaoka ${ }^{a}$ \\ ${ }^{a}$ Department of Internal Medicine, Seikeikai Hospital, Sakai, and ${ }^{b}$ Department of \\ Internal Medicine I, Osaka Medical College, Takatsuki, Japan
}

\section{Key Words}

Cryptococcal meningoencephalitis - Corticosteroid - Cryptococcus neoformans .

Immunocompetent host - Cryptococcal antigen titer - Paradoxical antifungal therapy-induced clinical deterioration

\begin{abstract}
We present a case of refractory Cryptococcus neoformans meningoencephalitis in an immunocompetent woman. Her clinical symptoms did not improve with 6 months of antifungal therapy, and MRI abnormalities, indicating severe meningeal and cerebral inflammation, persisted despite a decreasing cryptococcal antigen titer. The patient continued to deteriorate despite antifungal therapy, and her condition clearly improved following treatment with adjunctive corticosteroid. We postulate that the paradoxical antifungal therapy-related clinical deterioration was due to an immune response to cryptococcal organisms, which responded to corticosteroids. These observations provide rationale for a further evaluation of corticosteroids in the management of select cases of $C$. neoformans central nervous system infection.


Nakajima et al.: Refractory Cryptococcus neoformans Meningoencephalitis in an Immunocompetent Patient

\section{Introduction}

Cryptococcus neoformans commonly causes infection in immunocompromised hosts. However, $10-30 \%$ of the cryptococcal meningoencephalitis (CM) cases occur in HIVnegative patients $[1,2]$. The treatment of CM involves antifungal therapy and the management of intracranial pressure. In addition, reduced immunosuppressive therapy or HIV treatment is required to improve the immune status in cases with apparent immune deficiency. Combination therapy with amphotericin B and flucytosine has been recommended in previously published management guidelines for the treatment of cryptococcal infection of the central nervous system (CNS). Most immunocompetent patients can be successfully treated with this combination therapy within 6 weeks [3, 4]. However, some cases may be refractory and fulminant despite the recommended treatment. We present a case of CM in a previously healthy woman. Although her symptoms and magnetic resonance imaging (MRI) abnormalities did not improve with 6 months of antifungal therapy, she was successfully treated with an adjunctive corticosteroid.

\section{Case Presentation}

A 69-year-old immunocompetent woman presented with a 6-week history of loss of appetite, nausea, headache, and fever. She was initially referred to a local hospital, where systemic computed tomography and fluorodeoxyglucose positron emission tomography computed tomography findings were normal. Further, she was admitted to our hospital with severe headache and fever. The patient was a non-smoker and had no family history of any major illness. Clinical examination showed nuchal rigidity and a Glasgow Coma Scale (GCS) of 13/15 (eye movement 4, verbal 4, and motor 5). No deficits were observed on examining the cranial nerve and all four limbs.

MRI revealed patchy enhancements of the sulci in the brain cortex, cerebellar, and brainstem (fig. 1a, d; arrows). A lumbar puncture was performed, and the cerebrospinal fluid (CSF) showed 69 cells $/ \mathrm{mm}^{3}$ (38\% mononuclear cells), a protein concentration of $168 \mathrm{mg} / \mathrm{dl}$ (normal value: 15-45), and a glucose concentration of $2 \mathrm{mg} / \mathrm{dl}$ (normal value: 40-85). A positive India ink test indicated that the patient was positive for cryptococcal antigen $(1: 2,048)$, and CSF cultures were positive for C. neoformans var. neoformans. HIV serology was negative.

Liposomal amphotericin B (L-AMB; $5 \mathrm{mg} / \mathrm{kg}$ per day) and flucytosine $(25 \mathrm{mg} / \mathrm{kg}$ four times a day) were started. However, flucytosine was canceled to avoid the side effect of liver damage and was replaced with intrathecal amphotericin B. Furthermore, intravenous L-AMB and intrathecal amphotericin B were administered for 6 weeks. Treatment was then switched to voriconazole ( $8 \mathrm{mg} / \mathrm{kg}$ per day) for 1 month. Despite these treatments, MRI after 11 weeks of therapy showed an increased enhancement of the sulci and an enlarged ventricular system (fig. 1b, e). Follow-up CSF examination revealed a decrease in the cryptococcal antigen (1:512) and negative culture; however, pleocytosis (44 cells $\left./ \mathrm{mm}^{3}\right)$ and an elevated protein concentration $(155 \mathrm{mg} / \mathrm{dl})$ persisted. Moreover, her state of confusion had worsened with a GCS of 10/15 (eye movement 3, verbal 3, and motor 4). Therefore, treatment was switched to L-AMB ( $5 \mathrm{mg} / \mathrm{kg}$ per day) again on day 78 of admission. External ventricular drainage was performed on day 86 , and she underwent a placement of a permanent ventriculoperitoneal shunt on day 135. Although CSF examination revealed a further decrease in the cryptococcal antigen $(1: 128)$ and negative culture, MRI after 23 weeks of therapy showed no changes. Because her clinical symptoms had not improved, additional treatment 
Nakajima et al.: Refractory Cryptococcus neoformans Meningoencephalitis in an Immunocompetent Patient

with high-dose methylprednisolone (a 3-day course of $500 \mathrm{mg}$ methylprednisolone) was initiated on day 180 , followed by prednisolone $(20 \mathrm{mg})$ once daily. With this treatment, she recovered from impaired consciousness and febrile state, and the MRI abnormalities had disappeared on day 224 (fig. 1c, f).

Intravenous antifungal therapy was switched to fluconazole oral therapy (200 mg/day), and corticosteroids were gradually tapered. The patient experienced no relapse of meningoencephalitis during a 2-year follow-up period. She had normal cognitive function and could independently perform most activities of daily living; however, significant gait deficits persisted.

\section{Discussion}

A patient's immune status is considered an important factor in determining the pathogenesis of CM [5]. The present case demonstrates the immunomodulatory effects of $C$. neoformans infection. Most patients with CM have an immunosuppressed status (e.g., HIV infection, glucocorticoid or chemotherapy, or solid organ transplantation recipients), but our patient did not. HIV was ruled out by repeated serology testing. She was treated according to the Infectious Disease Society of America Guidelines [3]. Further, although flucytosine was canceled to avoid liver damage, she received intravenous L-AMB and intrathecal amphotericin B for 6 months. CSF examination showed decreases in the cryptococcal antigen titer. However, her clinical symptoms and MRI abnormalities persisted. The patient's persisting symptoms and subsequent response to adjunctive corticosteroid therapy were consistent with sustained meningeal inflammation that resolved with steroids. Meningeal inflammation appeared to persist despite 6 months of anti-fungal therapy and lumbar puncture evidence of resolving cryptococcal infection.

Glucuronoxylomannan is the principal constituent of the capsular material of $C$. neoformans [6] and exerts many immunosuppressive effects [7], including interference with phagocytosis [8], direct inhibition of T-cell responses [9], and a shift to a T helper 2 immune response with an intrathecal production of cytokines, such as interleukin 10 [10]. Moreover, it appears that antifungal therapy may reverse the glucuronoxylomannan-induced local immunosuppression in C. neoformans infection [10]. Previous reports showed that C. gattii CNS infection in an immunocompetent host, capable of mounting an inflammatory response, may lead to a paradoxical clinical deterioration due to an antifungal therapy-induced reduction in glucuronoxylomannan [10-12]. In these cases, corticosteroids provide definite benefit. After 6 months of antifungal therapy, our patient developed clinical and radiological signs of severe meningeal and cerebral inflammation despite a decreasing cryptococcal antigen titer. Because her condition clearly improved by subsequent adjunctive corticosteroid treatment, we hypothesize that this patient experienced clinical deterioration due to persisting meningeal inflammation, possibly precipitated by the effects of antifungal therapy on the organism. Furthermore, this case was characterized by high cryptococcal antigen titers in both serum and CSF. A large amount of glucuronoxylomannan can cause a severe local immunosuppression in CNS. In AIDS-related cryptococcal meningitis, antiretroviral therapy often causes severe inflammatory reactions, such as immune reconstitution inflammatory syndrome, in which corticosteroids are beneficial [13]. It appears that antifungal therapy can reverse the glucuronoxylomannan-induced local immunosuppression of similar inflammatory reactions.

The most comprehensive observational study of an increased intracranial pressure in AIDS-related cryptococcal meningitis due to $C$. neoformans showed an association between the treatment with adjunctive corticosteroids and a reduced survival [14]. The Infectious 
Nakajima et al.: Refractory Cryptococcus neoformans Meningoencephalitis in an Immunocompetent Patient

Disease Society of America has advised against the use of steroids for HIV-negative patients due to the lack of an established benefit. However, our case indicates that corticosteroids may be beneficial even in immunocompetent patients with $C$. neoformans CNS infection who do not respond to amphotericin B treatment although CSF culture becomes negative and in those who develop new or worsening focal inflammatory brain lesions on follow-up MRI.

In conclusion, we report the case of CM in an immunocompetent patient who showed paradoxical antifungal therapy-induced clinical deterioration related to an immune response to cryptococcal organisms and a marked clinical response to corticosteroids. These observations further support the evaluation of corticosteroids in the management of select cases of C. neoformans CNS infection, including investigations of the dosing and timing of corticosteroid regimens.

\section{Statement of Ethics}

The authors have no ethical conflicts to disclose.

\section{Disclosure Statement}

The authors state that they have no conflicts of interest.

\section{References}

1 Lui G, Lee N, Ip M, et al: Cryptococcosis in apparently immunocompetent patients. QJM 2006;99:143-151.

-2 Dubbioso R, Pappatà S, Quarantelli M, et al: Atypical clinical and radiological presentation of cryptococcal choroid plexitis in an immunocompetent woman. J Neurol Sci 2013;334:180-182.

-3 Saag MS, Graybill RJ, Larsen RA, et al: Practice guidelines for the management of cryptococcal disease. Infectious Diseases Society of America. Clin Infect Dis 2000;30:710-718.

4 Dismukes WE, Cloud G, Gallis HA, et al: Treatment of cryptococcal meningitis with combination amphotericin B and flucytosine for four as compared with six weeks. N Engl J Med 1987;317:334-341.

5 Kumari R, Raval M, Dhun A: Cryptococcal choroid plexitis: rare imaging findings of central nervous system cryptococcal infection in an immunocompetent individual. Br J Radiol 2010;83:14-17.

-6 Cherniak R, Sundstrom JB: Polysaccharide antigens of the capsule of Cryptococcus neoformans. Infect Immun 1994;62:1507-1512.

7 Vecchiarelli A: Immunoregulation by capsular components of Cryptococcus neoformans. Med Mycol 2000;38:407-417.

8 Chayakulkeeree M, Perfect J: Cryptococcosis. Infect Dis Clin North Am 2006;20:507-544.

-9 Yauch LE, Lam JS, Levitz SM: Direct inhibition of T-cell responses by the Cryptococcus capsular polysaccharide glucuronoxylomannan. PLoS Pathog 2006;2:e120.

10 Einsiedel L, Gordon DL, Dyer JR: Paradoxical inflammatory reaction during treatment of Cryptococcus neoformans var. gattii meningitis in an HIV-seronegative woman. Clin Infect Dis 2004;39:e78-e82.

11 Phillips P, Chapman K, Sharp M, et al: Dexamethasone in Cryptococcus gattii central nervous system infection. Clin Infect Dis 2009;49:591-595.

12 Lane M, McBride J, Archer J: Steroid responsive late deterioration in Cryptococcus neoformans variety gattii meningitis. Neurology 2004;63:713-714.

$\$ 13$ Longley N, Harrison TS, Jarvis JN: Cryptococcal immune reconstitution inflammatory syndrome. Curr Opin Infect Dis 2013;26:26-34.

14 Graybill JR, Sobel J, Saag M, et al: Diagnosis and management of increased intracranial pressure in patients with AIDS and cryptococcal meningitis. Clin Infect Dis 2000;30:47-54. 


\section{Case Reports in Neurology}
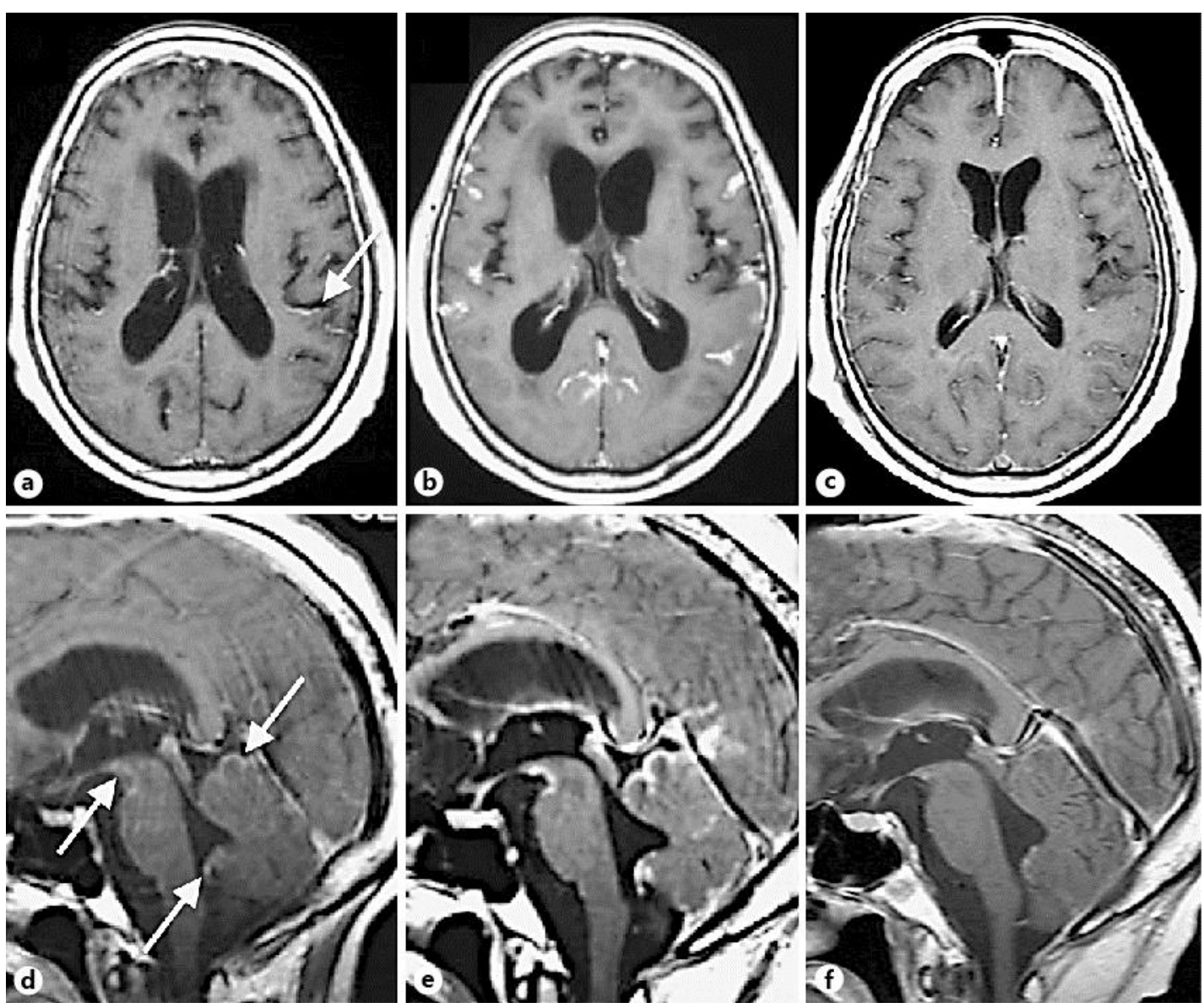

Fig. 1. Axial (a-c) and sagittal (d-f) T1-weighted gadolinium-enhanced MRI scans of the brain. At admission, patchy enhancements of the sulci in the brain cortex, cerebellar, and brainstem were revealed ( $\mathbf{a}, \mathbf{d}$; arrows). After 11 weeks of antifungal therapy, an increased enhancement of the sulci and an enlarged ventricular system were observed (b, e). These abnormalities disappeared at 32 weeks after treatment with an adjunctive corticosteroid $(\mathbf{c}, \mathbf{f})$. 